\title{
Sintilimab treatment for ureteral carcinoma following bladder cancer leads to leukopenia: a case report
}

\author{
Zhixian Zhong ${ }^{1 \#}$, Zhiying Wang ${ }^{2 \#}$, Yun $\mathrm{Li}^{3}$, Yi Zhong ${ }^{3}$ \\ ${ }^{1}$ Second Clinical Medical College Affiliated to Kunming Medical University, Kunming, China; ${ }^{2}$ College of Traditional Chinese Medicine, Tianjin \\ University of Traditional Chinese Medicine, Tianjin, China; ${ }^{3}$ Department of Oncology, Shanghai Traditional Chinese Medicine-Integrated Hospital, \\ Shanghai, China \\ \#These authors contributed equally to this work. \\ Correspondence to: Yun Li. Department of Oncology, Shanghai Traditional Chinese Medicine-Integrated Hospital, 230 Baoding Road, 6/F, Building 2, \\ Hongkou, Shanghai 200082, China. Email: 379103037@qq.com.
}

\begin{abstract}
Herein, we present a case report of sintilimab treatment for a patient with ureteral cancer reoccurring after bladder cancer, and exploration of the mechanism of adverse reactions from the aspects of intestinal flora and immunity. We have reported a case of leukopenia in a patient with recurrent ureteral cancer after bladder cancer who was treated with sintilimab. A 52-year-old Chinese man with a history of hypertension and diabetes presented with lower urinary tract symptoms, including painless hematuria, frequent and urgent urination, and micturition without pain. Computed tomography (CT) and 3-dimensional (3D) reconstruction suggested bladder space occupation, bladder cancer was pathologically confirmed after laser resection of the bladder tumor, which then recurred and was subject to reoperation. After 8 months, B-mode ultrasonography indicated left ureter occupation, and the patient began sintilimab immunotherapy according to the outcome of immunohistochemistry (IHC) and immune checkpoint inhibitor (ICI) evaluation. The patient was treated with sintilimab a total of 6 times. After the first treatment, the patient was in stable condition. The second treatment was discontinued due to renal insufficiency. The patient was then treated with renal and liver protection for 1.5 months, followed by 5 rounds of immunotherapy. After the sixth round of immunotherapy, the patient presented with leukopenia. In order to determine the causes of adverse reactions, we analyzed the changes of intestinal flora of patients before and after immunotherapy, and summarized the immune function indicators of patients during immunotherapy. The leucopenia induced by sintilimab may be related to intestinal flora and immunity.
\end{abstract}

Keywords: Papillary urothelial carcinoma; sintilimab; myelosuppression; leucopenia; case report

Submitted Nov 26, 2021. Accepted for publication Jan 30, 2022.

doi: $10.21037 / \mathrm{tau}-21-1146$

View this article at: https://dx.doi.org/10.21037/tau-21-1146

\section{Introduction}

The urinary system includes the kidneys, ureter, bladder, prostate, and urethra. According to different anatomical positions, it can be divided into the upper urinary tract and lower urinary tract. The upper urinary tract includes the kidneys and ureter, while the lower urinary tract includes the bladder, prostate, and urethra. Upper urothelial neoplasms are less common and ureteral neoplasms are even rarer, accounting for only a quarter of the total urinary neoplasms. The conventional treatment for ureteral cancer is surgery, followed by bladder perfusion, chemotherapy, radiotherapy, and immunotherapy. Immune checkpoint inhibitors (ICIs) have instigated a new era in the treatment of advanced or metastatic urothelial carcinoma, and the remarkable results achieved to date suggest that immunotherapy may be the future paradigm for ureteral cancer (1). However, immunerelated adverse reactions caused by ICIs greatly affect the clinical treatment effect and the quality of life of patients, among which marrow suppression with leukopenia as the initial manifestation is one of the common adverse reactions. 

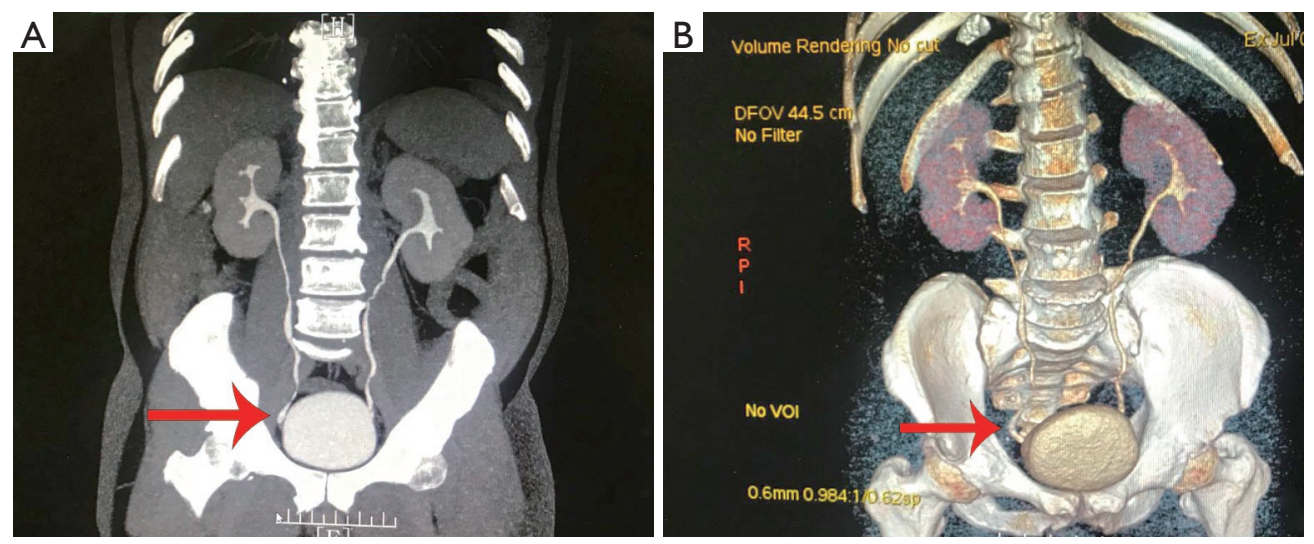

Figure 1 Radiomics data of patients before surgery. (A) The morphology, size, and density of both pelvis calices are normal, and bilateral ureteral development is unobstructed without stenosis or obstruction. The bladder is well filled, and the posterior wall of the bladder is thickened near the right ureter entrance (at the red arrow), with a diameter of about $7.8 \mathrm{~mm}$. No obvious dilatation or effusion is obvious in the right upper urinary tract. (B) The posterior wall of the bladder is thickened near the right ureteral entrance (at the red arrow). Malignant tumor is considered. No obvious dilatation or effusion is seen in the right upper urinary tract. The rest showed no obvious abnormality.

Adverse reaction to immunotherapy is one of the challenges to the treatment of ureteral cancer.

Sintilimab is a humanized IgG4 monoclonal antibody that binds to programmed cell death receptor-1 (PD-1), thereby blocking PD-1 from interacting with its ligands (PD-L1 and PD-L2), thereby contributing to the restoration of endogenous anti-tumor $\mathrm{T}$ cell responses. Herein, we have focused on the immune-related adverse events of sintilimab treatment for a patient with ureteral cancer following bladder cancer, and explored the mechanism of adverse reactions from the aspects of intestinal flora and immunity.

We present the following article in accordance with the CARE reporting checklist (available at https://tau. amegroups.com/article/view/10.21037/tau-21-1146/rc).

\section{Case presentation}

A 52-year-old Chinese man presented painless hematuria, frequent urination, and urgent urination without obvious inducement in June 2018. In July 2018, he went to Shanghai General Hospital for treatment, and computed tomography (CT) examination suggested bladder occupation (Figure 1A), which was confirmed by a 3-dimensional (3D) reconstruction (Figure 1B). On 12 July 2018, the patient underwent transurethral laser resection of the plasma bladder tumor. Postoperative pathology revealed papillary urothelial carcinoma, some of high grade, without obvious infiltration. On 13 December 2018, the patient underwent transurethral plasma bladder tumor resection under general anesthesia. Pathology: a total of 6 single tumor cells and 0 groups of microembolic cells were detected. The patient underwent B-ultrasound reexamination in Shanghai General Hospital after bladder cancer in August 2019: the left ureter was occupied. On 5 September 2019, the patient underwent ureteroscopic resection of left ureteral tumor plus laparoscopic radical resection of left ureteral carcinoma, the patient received 1 unit of blood during the operation. Postoperative pathology (Pathology Number: 19-15458) revealed the following: left kidney + full length of left ureter + partial bladder tissue excision specimen: left ureteral papillary urothelial carcinoma of high grade with invasion of lamina propria and mass size of $7 \mathrm{~cm} \times 3 \mathrm{~cm}$ $\times 1.2 \mathrm{~cm}$. No clear neurovascular invasion was observed. Partial renal pelvis epithelium hyperplasia with atypia. There was no tumor involvement in the broken end of ureter, short end of renal hilar vessels, or renal tissue. The detection of tumor markers was as follows: "C, F": CK7+, CK20+, CK5/6-, P63+, Ki67 (30-40\%+). The findings of postoperative pathology (Pathology Number: 19-15475) were as follows: ureteral mass: no tumor involvement at the left ureteral terminal margin; no tumor involvement at the left ureterovesical margin; papillary urothelial carcinoma (mass at the left end of the ureter) of high grade. On 18 September 2019, the efficacy evaluation of ICIs was carried out in the hospital, and the results are as follows: (I) tumor mutation burden $(\mathrm{TMB})=300$. Mutations $/ \mathrm{Mb}$, total number of somatic edited mutations: 42; (II) conclusion: it may be 
Table 1 Changes in blood routine of patients treated with sintilimab

\begin{tabular}{|c|c|c|c|c|c|}
\hline Date & WBC count $\left(\times 10^{9} / \mathrm{L}\right)$ & Neutrophil count $\left(\times 10^{9} / \mathrm{L}\right)$ & RBC count $\left(\times 10^{12} / L\right)$ & Hemoglobin (g/L) & Platelet count $\left(\times 10^{9} / \mathrm{L}\right)$ \\
\hline 2019/11/1 & 10.60 & 6.79 & 4.21 & 129 & 255 \\
\hline 2019/11/7 & 8.90 & 5.79 & 4.12 & 124 & 235 \\
\hline 2019/12/19 & 7.20 & 4.98 & 4.4 & 133 & 198 \\
\hline $2020 / 2 / 5$ & 3.90 & 2.70 & 4.04 & 122 & 156 \\
\hline $2020 / 2 / 26$ & $2.60 \downarrow$ & $1.40 \downarrow$ & $4.04 \downarrow$ & 123 & 193 \\
\hline $2020 / 3 / 17$ & $2.60 \downarrow$ & Miss & $4.18 \downarrow$ & 128 & 172 \\
\hline $2020 / 4 / 14$ & $1.30 \downarrow$ & $0.36 \downarrow$ & 4.38 & 135 & 132 \\
\hline
\end{tabular}

WBC count, neutrophil count and RBC count drops over time. WBC, white blood cell; RBC, red blood cell; Miss, the count is too low to read.

sensitive to ICI treatment. Therefore, he received $200 \mathrm{mg}$ of the first sintilimab immunotherapy in our hospital on 14 October 2019. After treatment, his general condition was satisfactory, and he was discharged after stabilization. On 31 October 2019, due to renal insufficiency, he did not receive the second program of sintilimab treatment, and was discharged from Shanghai General Hospital for treatment. After long-term treatment, he took Jinshuibao tablets (4 tablets, TID) and compound ketoic acid tablets (4 tablets, TID) for renal protection. The reexamination of liver function on 19 December 2019 showed increased alanine transaminase (ALT) and aspartate transaminase (AST), suggesting abnormal liver function. On 20 December 2019, 9 January 2020, 6 February 2020, 27 February 2020, and 19 March 2020, the patient received 5 courses of sintilimab $200 \mathrm{mg}$ immunotherapy, during which the leukocyte and neutrophils showed progressive decline, and the red blood cells, hemoglobin, and platelets were at a low level (Table 1), whereby leukopenia was clinically diagnosed. On 14 April 2020, the white blood cell (WBC) count was $1.3 \times 10^{9} / \mathrm{L}$, and the neutrophil count was $0.36 \times 10^{9} / \mathrm{L}$. The patient was diagnosed with leukopenia and given human granulocyte colony stimulating factor (CSF) injection (75 g, subcutaneous injection, bid) and dexamethasone tablet $(0.75 \mathrm{mg}, \mathrm{QD})$. We used $16 \mathrm{sRNA}$ count to analyze the intestinal flora of the patient before and after immunotherapy. The patient had a history of hypertension for 10 years, and had taken olmesartan $(20 \mathrm{mg}, \mathrm{QD})$ and metoprolol tartrate (50 mg, QD) for an extended period. He also had a history of diabetes for many years, with long-term use of calcium hydroxybenzenesulfonate capsules $(500 \mathrm{mg}$, BID). The patient's father had died of esophageal cancer.
All procedures performed in this study were in accordance with the ethical standards of the institutional and/or national research committee(s) and with the Helsinki Declaration (as revised in 2013). Written informed consent was obtained from the patient for publication of this case report and accompanying images. A copy of the written consent is available for review by the editorial office of this journal.

\section{Discussion}

Immune-related adverse events due to excessive activation of $\mathrm{T}$ cells in ICI therapy is associated with immune and blood interactions, and developed a reporting system, which mainly includes four categories: autoimmune hemolytic anemia, immune thrombocytopenia, neutropenia, and aplastic anemia and pure red cell anemia (2). Moreover, neutropenia is a rare immune-related adverse reaction that often occurs at the same time as immune leukopenia. As a programmed cell death protein 1 (PD-1) inhibitor, sintilimab has excellent clinical efficacy in the treatment of a variety of solid tumors, and was officially approved in China on 24 December 2018 (3). The ORIENT1 trial showed that sintilimab was safe in treating refractory Hodgkin's lymphoma, and 1 of the 96 participants developed thrombocytopenia, but none developed leukopenia or neutropenia (4).

In this case, the WBC and neutrophil granulocytes showed progressive decline after treatment with 6 sintilimab at $200 \mathrm{mg}$. The white blood cell count decreased by $86.73 \%$ during the six months of treatment, and the Neutrophil count decreased by $93.84 \%$. Sintilimab is an antibody-building drug that may cause leukopenia and neutrophilia by a mechanism similar to that of immune- 
Table 2 Changes of immune indexes in patients treated with sintilimab

\begin{tabular}{|c|c|c|c|c|c|c|}
\hline Date & $\begin{array}{l}\text { NK cell count } \\
(/ \mu \mathrm{L})\end{array}$ & $\begin{array}{c}\text { NK cell } \\
\text { percentage }(\%)\end{array}$ & $\begin{array}{l}\text { Total T lymphocyte } \\
\text { count }(/ \mu \mathrm{L})\end{array}$ & $\begin{array}{l}\text { Lymphocyte count } \\
(/ \mu \mathrm{L})\end{array}$ & $\begin{array}{c}\text { Cytotoxic } T \\
\text { lymphocyte count }(/ \mu \mathrm{L})\end{array}$ & $\begin{array}{c}\text { Induced T lymphocyte } \\
\text { count }(/ \mu \mathrm{L})\end{array}$ \\
\hline 2019/10/11 & 765 & 27.13 & 1,596 & 2,783 & 553 & 931 \\
\hline $2019 / 11 / 1$ & 781 & 27.23 & 1,640 & 2,835 & 549 & 949 \\
\hline $2019 / 11 / 28$ & 163 & 13.07 & 836 & 1,199 & 236 & 534 \\
\hline $2020 / 2 / 6$ & $3.00 \downarrow$ & $0.40 \downarrow$ & $507 \downarrow$ & $739 \downarrow$ & $156 \downarrow$ & $293 \downarrow$ \\
\hline
\end{tabular}

On 26 February 2020, the patient experienced a decline in immune function at the fourth immunotherapy session. NK, natural killer.

mediated leukopenia. When the drug binds to the cell membrane, the membrane antigen epitopes are changed, and autoantibodies are stimulated to form, which directly destroys the granulocytes in peripheral blood and bone marrow, thus inhibiting the formation of granulocytes and monoclonal hematopoietic progenitors. The treatment of immunoneutropenia is challenging and the optimal longterm treatment has not been determined. In order to prevent the patients from experiencing serious infection during the follow-up treatment, several methods have been proposed to improve the level of neutrophils (5). Due to the limited effect of steroids and the temporary effectiveness of intravenous immunoglobulin in $50 \%$ of patients (6), the clinical efficacy evaluation was poor. For primary autoimmune neutropenia, granulocyte CSF is the most effective treatment, it can stimulate progenitor cells proliferation and maturation, increase production and release of mature neutrophils, foster neutrophils induced matrix, reduce neutrophil apoptosis, and cut neutral grain of cell membrane antigen expression (7). However, the effect of granulocyte CSF is temporary and undetermined, and usually recurs when treatment is discontinued. How to maintain the role of granulocyte CSF effectively and persistently remains to be further studied in basic experiments and clinical trials.

Cytotoxic $\mathrm{T}$ lymphocytes usually recognize cancer cells or cells infected with pathogens, which is why we live to adulthood. However, when overactive immune cells are continuously summoned to tumor cells or infected sites, they fail and can no longer be assigned to the "invader" (8). In this case, after 6 sintilimab treatments of $200 \mathrm{mg}$, the cytotoxic $\mathrm{T}$ lymphocytes showed progressive decline (Table 2), and leukopenia may be related to $\mathrm{T}$ cell failure. In the case of lymphocytosis, T cells will continue to divide to maintain peripheral blood pool volume (9).
In addition, there were 137 kinds of the same bacteria before and after immunotherapy through 16sRNA analysis; however, 22 kinds of bacteria disappeared after treatment, and 35 new bacteria appeared, indicating that the immunotherapy with sintilimab had a regulatory effect on the intestinal flora (Figure 2A, Table 3). After patients had experienced adverse reactions after immunotherapy, the general trend of bacterial flora changes was that opportunistic pathogens decreased and probiotics increased (Figure 2B). It has been reported in literature (10) that bone marrow suppression is a side effect associated with many antibiotics, especially in the case of long-term use of antibiotics. Recent advances in our understanding of homeostatic hematopoiesis have allowed us to explore in detail the effects of antibiotics on hematopoietic progenitor cells using mouse models. Mice treated with antibiotics developed anemia, thrombocytopenia, and leukopenia, and flow cytometry analysis of peripheral blood showed pan-lymphopenia. Bone marrow progenitor cell analysis showed that all subtypes of hematopoietic stem cells and pluripotent progenitors were depleted. Granulocytes and $\mathrm{B}$ cells decreased while $\mathrm{CD} 8^{+} \mathrm{T}$ cells increased. No reduction in progenitor cell activity was observed when the cells were incubated directly with antibiotics, suggesting that these effects were indirect. Hematopoietic function was associated with significant contraction of fecal flora, and was partly saved by fecal flora metastasis. In addition, mice raised under aseptic conditions showed hematopoietic abnormalities similar to those seen in antibiotic treated mice, while antibiotic treatment did not cause additional abnormalities in sterile mice.

Intestinal bacteria may affect circulatory WBC counts by influencing either their sources in the bone marrow, or their cytokine profiles and proliferation rates in the 
A
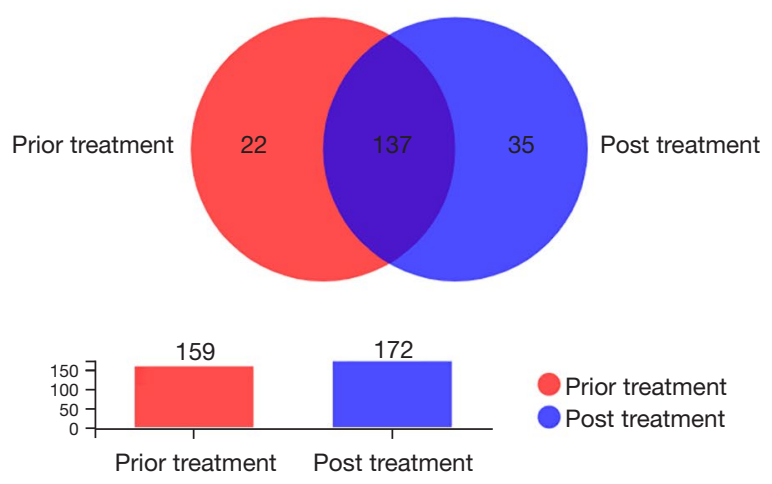

B

Community barplot analysis

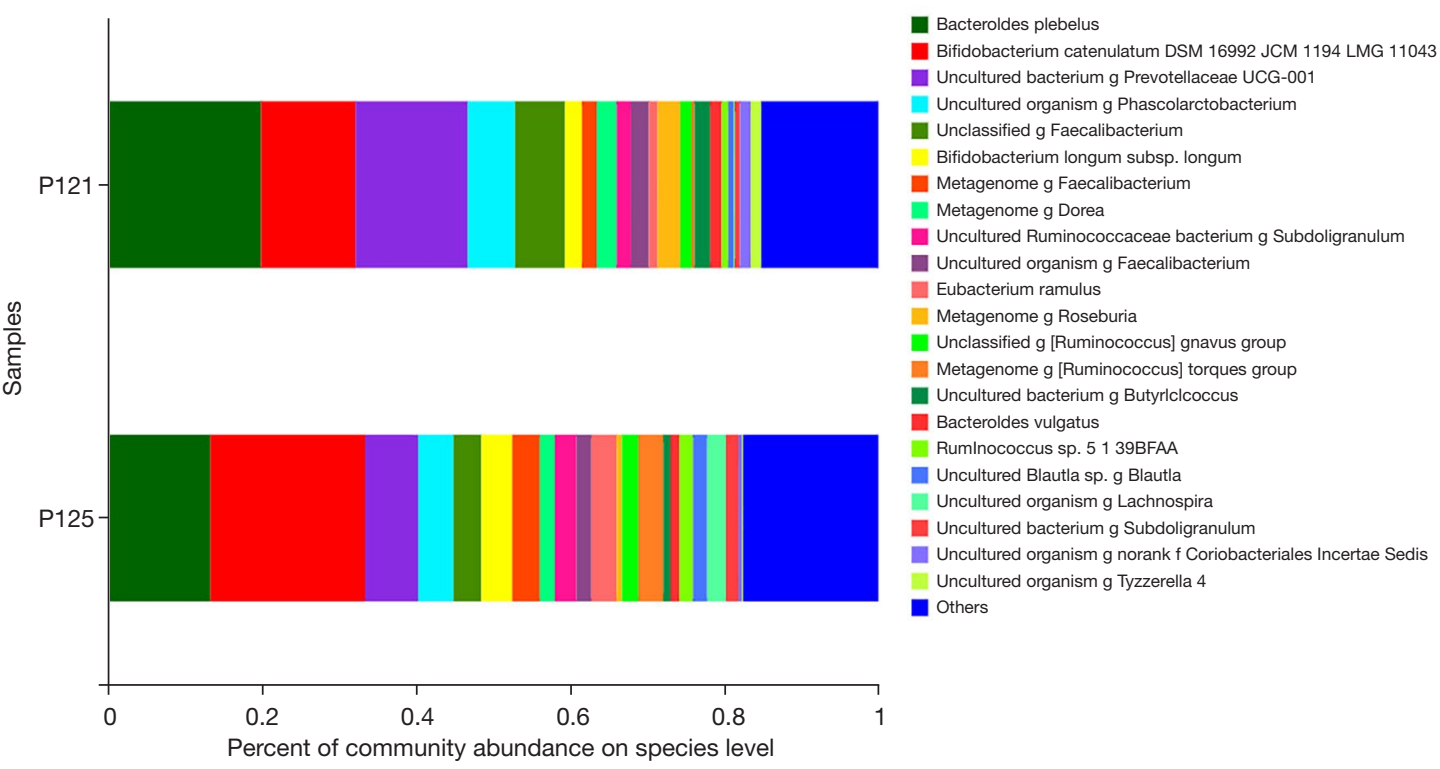

Figure 2 The intestinal flora of the patients before and after immunotherapy. (A) Venn diagram of intestinal flora changes before and after immunotherapy; (B) percent of community abundance on species level. P121 and P125 respectively means prior treatment and posttreatment sample numbers of the patient in our clinical trial.

Table 3 Changes of intestinal flora before and after immunotherapy in the first 6 species

\begin{tabular}{lccc}
\hline Name & Type & Pretreatment proportion (\%) & Posttreatment proportion (\%) \\
\hline Bacteroldes & Conditioned pathogen & 19.88 & 13.30 \\
Bifidobacterium_catenulatum & Probiotics & 12.26 & 20.09 \\
Prevotellaceae_UCG-001 & Conditioned pathogen & 14.55 & 6.89 \\
Phascolarctobacterium & Conditioned pathogen & 6.14 & 4.57 \\
Unclassified g Faecalibacterium & Probiotics & 6.48 & 3.62 \\
Bifidobacterium_longum & Probiotics & 2.21 & 3.99 \\
\hline
\end{tabular}

After immunotherapy, the abundance of probiotics in the intestinal flora of the patients increased, while the abundance of opportunistic pathogens decreased. 


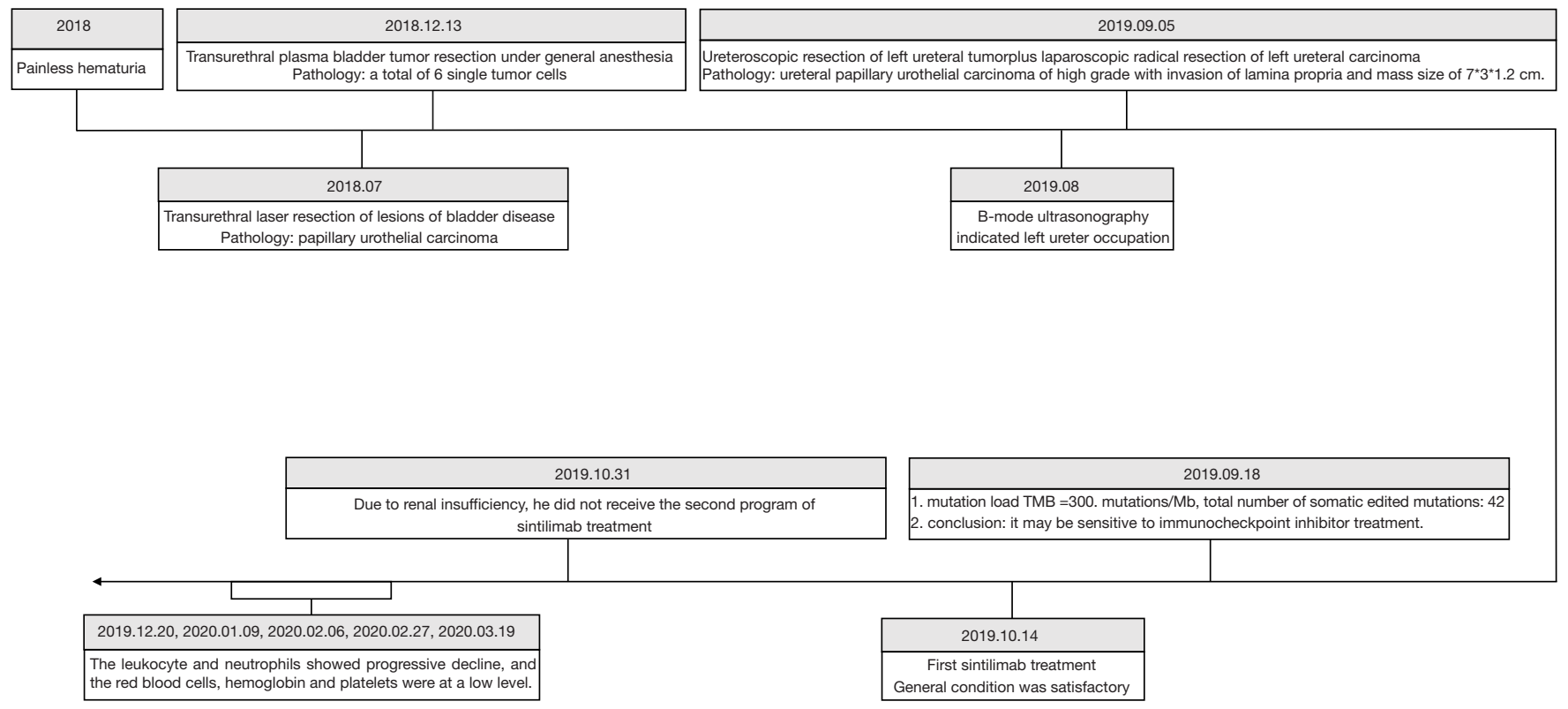

Figure 3 Depiction of important dates and times in this case.

blood, or their sinks in different organs, or all. Leukocytes include neutrophils, eosinophils, basophils, lymphocytes and monocytes. In this case, the patient showed a rapid decrease in neutrophil count. It has been confirmed that Rothia (conditioned pathogen) and Clostridium sensu stricto (pathogen) are significantly correlated with neutrophil decline (11). Interestingly, the case also concluded that the decline of neutrophils may be associated with a down-regulation in the abundance of conditioned pathogens. Therefore, the regulation of intestinal flora also has a certain influence on the occurrence of bone marrow suppression, but the specific mechanism by which immunotherapy induces the occurrence of adverse reactions of bone marrow suppression through the regulation of intestinal flora needs to be further explored.

According to the clinical experience of immunotherapy for malignant tumors in our department, we usually evaluate the immune status and systemic body function of patients before they receive immunotherapy, including blood routine, liver and kidney function, immune indicators, inflammatory factors, intestinal flora, intestinal barrier (such as DAO, ET, D-LA). For patients with high risk factors of immunotherapyrelated adverse reactions, we do not recommend the use of immune checkpoint inhibitors. At the same time, we suggest patients to take TCM granules during immunotherapy to achieve the effect of synergism and toxicity reduction. However, it is regrettable that the patient in this case refused to accept TCM treatment, and there was no clear abnormality in the preliminary examination indicators, which may be related to the lack of unified standards for the results of intestinal flora at present. In addition, we suggest that patients avoid using cellular immunomodulators (such as thymosin, etc.) during immunotherapy and actively supplement probiotics (such as Clostridium butyricum Tablets, etc.), which can effectively prevent the occurrence of adverse reactions of immunotherapy.

Finally, intestinal flora has been proven to play an important role in the tumorigenesis and prognosis of tumors, and it also can affect tumor response to immunotherapy (12-15). Sulforaphane has been shown to prevent chemically-induced bladder cancer in mice by regulating the composition of intestinal flora, repairing physiological damage to the intestinal barrier, reducing inflammation and immune response. Sulforaphane could normalize the dysregulation of intestinal microflora in n-butyl-n-(4hydroxybutyl-Nitrosamine-induced bladder cancer mice, and significantly increase the number of Bacteroides vulnerable and Clostridium cluster I. Sulforaphane also increased colon butyric acid levels in mice (16). In addition, intestinal flora can affect the occurrence and development of urinary tumor, urinary tract flora plays an equal role, which will be further explored in the future. In summary, it took more than 2 years from symptom onset to treatment termination (Figure 3). The patient believed that the treatment was 
effective in reducing the probability of tumor recurrence, but due to serious adverse reactions, he chose cystoscopy for regular outpatient follow-up. Until the submission time, he has not exhibited recurrence and metastasis, and he is still alive at the time of publication with regular outpatient follow-up.

\section{Acknowledgments}

Funding: This work was supported by Clinical medicine project of Shanghai "Science and Technology Innovation Action Plan" in the field of clinical medicine to Yi Zhong (19401971600); Innovative project for graduate student of Traditional Chinese Medicine of Shanghai University to Shiying Li (Y2021063). Shiying Li is the master student in 2020 class of Yi Zhong.

\section{Footnote}

Reporting Checklist: The authors have completed the CARE reporting checklist. Available at https://tau.amegroups.com/ article/view/10.21037/tau-21-1146/rc

Conflicts of Interest: All authors have completed the ICMJE uniform disclosure form (available at https://tau.amegroups. com/article/view/10.21037/tau-21-1146/coif). The authors have no conflicts of interest to declare.

Ethical Statement: The authors are accountable for all aspects of the work in ensuring that questions related to the accuracy or integrity of any part of the work are appropriately investigated and resolved. All procedures performed in this study were in accordance with the ethical standards of the institutional and/or national research committee(s) and with the Helsinki Declaration (as revised in 2013). Written informed consent was obtained from the patient for publication of this case report and accompanying images. A copy of the written consent is available for review by the editorial office of this journal.

Open Access Statement: This is an Open Access article distributed in accordance with the Creative Commons Attribution-NonCommercial-NoDerivs 4.0 International License (CC BY-NC-ND 4.0), which permits the noncommercial replication and distribution of the article with the strict proviso that no changes or edits are made and the original work is properly cited (including links to both the formal publication through the relevant DOI and the license).
See: https://creativecommons.org/licenses/by-nc-nd/4.0/.

\section{References}

1. Shao IH, Chang YH, Pang ST. Recent advances in upper tract urothelial carcinomas: From bench to clinics. Int J Urol 2019;26:148-59.

2. Zhuang J, Du J, Guo X, et al. Clinical diagnosis and treatment recommendations for immune checkpoint inhibitor-related hematological adverse events. Thorac Cancer 2020;11:799-804.

3. Liu X, Yi Y. Recent updates on Sintilimab in solid tumor immunotherapy. Biomark Res 2020;8:69.

4. Shi Y, Su H, Song Y, et al. Safety and activity of sintilimab in patients with relapsed or refractory classical Hodgkin lymphoma (ORIENT-1): a multicentre, single-arm, phase 2 trial. Lancet Haematol 2019;6:e12-9.

5. Bigot W, Viallard JF, Sellam J, et al. Efficacy and safety of rituximab for autoimmune neutropenia: a French case series and literature review. Rheumatology (Oxford) 2020;59:2637-40.

6. Sicre de Fontbrune F, Moignet A, Beaupain B, et al. Severe chronic primary neutropenia in adults: report on a series of 108 patients. Blood 2015;126:1643-50.

7. Capsoni F, Sarzi-Puttini P, Zanella A. Primary and secondary autoimmune neutropenia. Arthritis Res Ther 2005;7:208-14.

8. Mognol GP, Spreafico R, Wong V, et al. Exhaustionassociated regulatory regions in CD8+ tumor-infiltrating T cells. Proc Natl Acad Sci U S A 2017;114:E2776-85.

9. Saidakova EV, Shmagel KV, Korolevskaya LB, et al. Lymphopenia-induced proliferation of CD4 T-cells is associated with CD4 T-lymphocyte exhaustion in treated HIV-infected patients. Indian J Med Res 2018;147:376-83.

10. Josefsdottir KS, Baldridge MT, Kadmon CS, et al. Antibiotics impair murine hematopoiesis by depleting the intestinal microbiota. Blood 2017;129:729-39.

11. Schluter J, Peled JU, Taylor BP, et al. The gut microbiota is associated with immune cell dynamics in humans. Nature 2020;588:303-7.

12. Viaud S, Saccheri F, Mignot G, et al. The intestinal microbiota modulates the anticancer immune effects of cyclophosphamide. Science 2013;342:971-6.

13. Peuker K, Muff S, Wang J, et al. Epithelial calcineurin controls microbiota-dependent intestinal tumor development. Nat Med 2016;22:506-15.

14. Yu LX, Schwabe RF. The gut microbiome and liver cancer: mechanisms and clinical translation. Nat Rev 
Gastroenterol Hepatol 2017;14:527-39.

15. Ternák G, Berényi K, Sümegi A, et al. Antibiotic Consumption Patterns in European Countries May Be Associated with the Incidence of Major Carcinomas. Antibiotics (Basel) 2020;9:643.

Cite this article as: Zhong Z, Wang Z, Li Y, Zhong Y. Sintilimab treatment for ureteral carcinoma following bladder cancer leads to leukopenia: a case report. Transl Androl Urol 2022;11(2):277-284. doi: 10.21037/tau-21-1146
16. He C, Huang L, Lei P, et al. Sulforaphane Normalizes Intestinal Flora and Enhances Gut Barrier in Mice with BBN-Induced Bladder Cancer. Mol Nutr Food Res 2018;62:e1800427. 